\title{
Wie Frauen im Rollstuhl Schwangerschaft, Geburt und Mutterrolle meistern
}

Anja Stamm, Ute Lange

„Waaas - ihr wollt Kinder bekommen?“ Bisweilen treffen Frauen im Rollstuhl auf Vorbehalte, wenn sie sich mit ihrem Partner für ein Kind entscheiden. Auch Hebammen und Geburtshelfer sind vielfach nur rudimentär auf Schwangere mit Querschnittlähmung vorbereitet. Dabei gibt es keinen Grund für Berührungsängste. Potenzielle Risiken sind erforscht und Empfehlungen für die Betreuung und Behandlung verfügbar. DIE HEBAMME hat Frauen mit Rückenmarkschädigung befragt, wie ihre Schwangerschaft und Geburt verlaufen ist und wie ihr Leben mit Baby und Handicap gelingt.

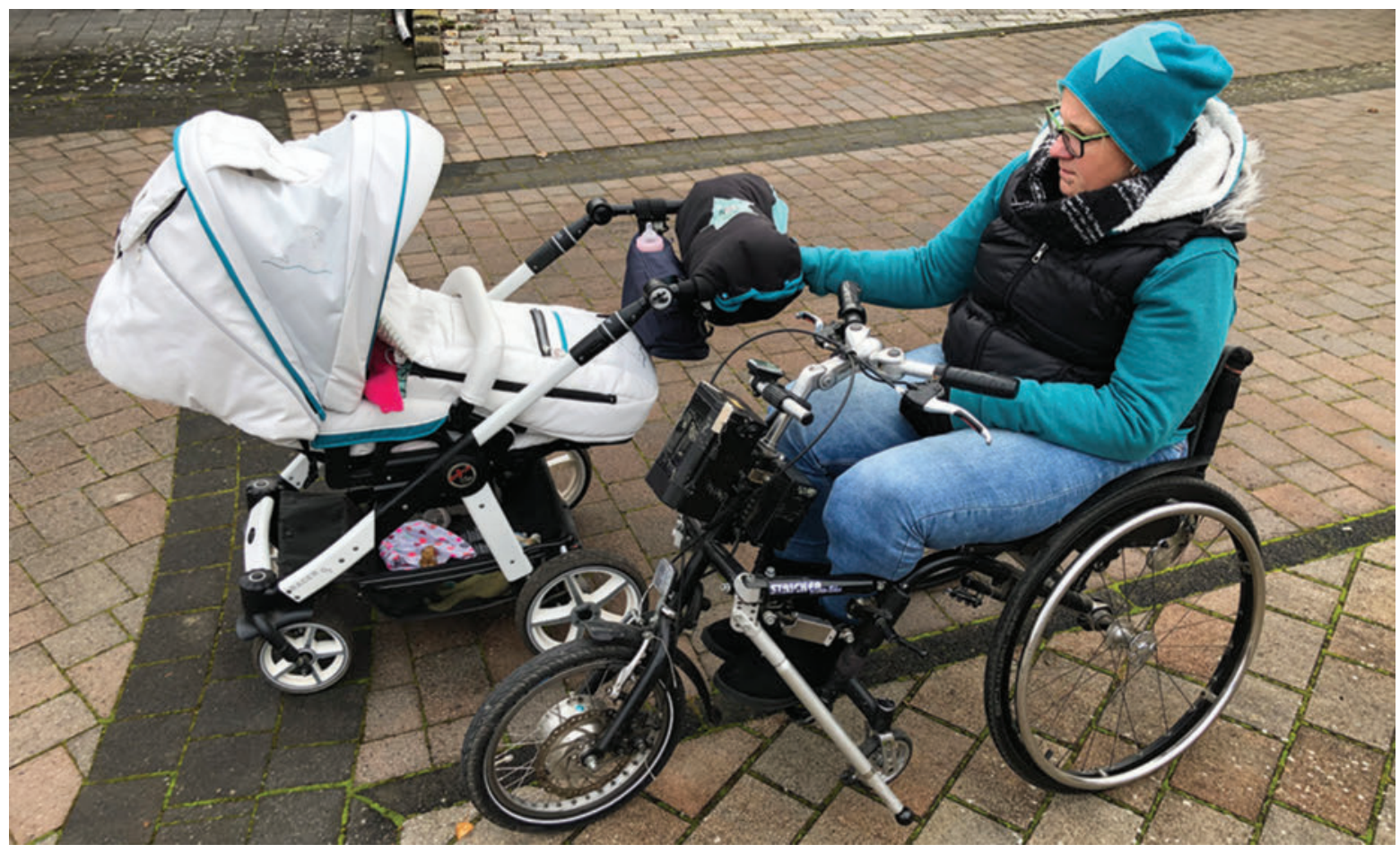

- Abb. 1 Spaziergang mit dem Kinderwagen und dem Elektrofahrrad: Verschiedene Hilfsmittel und clevere Ideen erleichtern das Leben mit Baby und Querschnittlähmung. (Foto: privat) [rerif]

\section{Den Kinderwunsch verwirklichen}

„ „hrem Untermieter ist es egal, ob Sie Sie im Rollstuhl sitzen." Bei diesen Worten ihres Gynäkologen plumpste Anna B. ( (*Name von der Redaktion geändert) ein Stein vom Herzen. Zwar hatten sie und ihr Partner sich sehnlichst ein Baby gewünscht. Doch bevor sie die Verhütung wegließ, wollte sie genauer wissen, ob ihre körperliche
Einschränkung eine Schwangerschaft oder das Kind negativ beeinflussen könnte. Seit einem Autounfall ist die heute 58-Jährige durch eine Rückenmarkschädigung im Bereich der Halswirbelsäule hochgradig gelähmt. Sie betätigt einen Elektrorollstuhl und ist damit trotz eingeschränkter Fingerfunktion mobil. Ihren Kinderwunsch verwirklichte sie mit 33 Jahren zum ersten Mal. 
„Mein Gynäkologe war nicht spezialisiert auf das Gebiet, hat aber gesagt, es ist nicht von Belang, ob ich im Rollstuhl sitze oder nicht. Das hat mich schon mal beruhigt. Dementsprechend bin ich da ganz blauäugig drangegangen. Ich wurde ziemlich bald schwanger. Das war für mich völliges Neuland, diese körperlichen Symptome. Starke Übelkeit und Schwäche, Müdigkeit ... Das ist schon anders, wenn man im Rollstuhl sitzt und auf Hilfe angewiesen ist. Weil man sich nicht mal einfach so hinlegen kann. Und wenn einem übel wird, kann man sich nicht einfach über die Toilette hängen. Man muss sehen, dass man immer eine Schüssel parat hat. Die ersten drei Monate mit den typischen Schwangerschaftssymptomen waren eben anstrengend. Da ich auch eine Blasenlähmung habe, hatte ich auch mit Blasenentzündungen zu tun. Die wurden aber nicht behandelt. Ich habe versucht, sie mit viel Trinken in den Griff zu bekommen, ohne Antibiotikum.“

\section{Öfter mal nach dem Kind schauen}

Anna B. ${ }^{*}$ hatte nach eigenen Worten in ihrer Schwangerschaft „sehr viel Glück“. Es traten trotz der Bewegungseinschränkungen keine außergewöhnlichen Symptome auf, die sie hätte befürchten können, wie z. B. extreme Wassereinlagerungen oder eine Thrombose. „Ich bin gut durch die Schwangerschaften gekommen." Ihr erstes Kind ist heute 24 Jahre alt, das zweite 13.

Um sicher zu gehen, dass wirklich alles in Ordnung ist, verkürzte die werdende Mutter die Abstände zwischen den Vorsorgeuntersuchungen. „Ich habe darum gebeten. Denn durch die verminderte Sensibilität hat man nicht so ein gutes Gefühl dafür, ob alles noch richtig ist. Das birgt eine große Unsicherheit, ob man vielleicht etwas versäumt oder nicht spürt. Bei der ersten Schwangerschaft hatte ich plötzlich so ein Ziehen und Stechen. Ich bin total panisch geworden, weil ich das nicht kannte. Ich hab nur gedacht: ,Hilfe, ich hab jetzt eine Fehlgeburt!' Ich war nervlich auch ein bisschen fertig und konnte das nicht einschätzen. Dann hab ich mit meinem Gynäkologen Rücksprache gehalten. Der hat mich dann beruhigt und gesagt, dass das alles völlig normal ist, dass die Bänder sich dehnen und dass es solche Beschwerden gibt. Aber ich könnte durchaus öfter zur Kontrolle kommen, damit ich Sicherheit habe. Dadurch war ich ein bisschen beruhigter. Ansonsten habe ich das Körpergefühl in der Schwangerschaft als sehr wohltuend empfunden. Ich hab mich ein bisschen wie ein vollgegessener Buddha gefühlt (lacht) ... so in sich ruhend. Ich war ganz zurückgenommen auf mich und mein Kind. Eine sehr schöne Erfahrung. Dieses Insich-hineinspüren-ich habe ja Kindsbewegungen gespürt. Das war schon ein Glücksgefühl. Das hat aber sicher auch eine Schwangere, die nicht im Rollstuhl sitzt."

\section{Den Geburtstermin planen}

Anna B.* brachte beide Kinder mit einem geplanten Kaiserschnitt zur Welt, der auf zwei Wochen vor dem errechneten Geburtstermin datiert wurde, um keine Spontangeburt zu riskieren. Heute weiß sie, warum von einer physiologischen Geburt abzuraten war: „Das geht bei einer so hohen Lähmung, wie ich sie habe, sehr stark auf den Kreislauf. Puls und Herzschlag erhöhen sich drastisch und der Blutdruck steigt." Als sie vor über 20 Jahren der Sectio zustimmte, wurde sie nicht ausgiebig aufgeklärt: „Die Ärzte waren nicht groß geschult, was Querschnittlähmung und Geburt angeht. Für die stand fest: natürlich Kaiserschnitt! Damit war ich völlig zufrieden. Ich konnte mir auch nicht vorstellen, wie das anders funktionieren sollte, auch aufgrund der starken Spastik." Beide Kaiserschnittgeburten verliefen komplikationslos. „Ich hatte keine frühzeitigen Wehen oder Ähnliches. Ich kann das sagen, denn vor dem zweiten Kind hatte ich eine Fehlgeburt, bei der ich auch Wehen hatte. Daher weiß ich jetzt, wie sich das anfühlt.“

\section{Den Rat der Hebamme genutzt}

In der ersten Schwangerschaft hatte Anna B.* eine Hebamme, die sie beriet und begleitete. „Sie kam zu Aufklärungsgesprächen und hat z.B. erklärt, wie ein Kaiserschnitt vor sich geht, was die Aufgabe des Vaters dabei sein kann, wie es danach weitergeht usw. Nach dem Kaiserschnitt ist die Hebamme regelmäßig zur Nachsorge gekommen. Eigentlich gab es aufgrund meiner Querschnittlähmung überhaupt keine Besonderheiten. Bei mir ist ja alles so problemlos abgelaufen. Ich hatte keine Probleme, dass die Narbe verheilt oder sich die Gebärmutter zurückbildet. Ich habe gestillt, nicht sehr lange, aber auch das hat ganz gut funktioniert. Ich kann wirklich nichts Schlimmes berichten. Mein Gynäkologe hat mir ebenfalls Mut gemacht. Sonst hätte ich wohl kein zweites Kind bekommen. Ich hatte beide Male Glück."

\section{KONTAKT \\ Hilfreiche Anlaufstellen für Eltern mit Behinderung}

Die besten Empfehlungen für Eltern mit Behinderung haben Eltern, die über eigene Erfahrung dazu verfügen. Kontakte und Informationen gibt es u. a. unter diesen Internetadressen:
Bundesweite Angebote
- www.lewu.de
- www.familienratgeber.de
- www.der-querschnitt.de
Internationale Angebote
- www.paraforum.ch
- www.disabledparents.net 
- www.apparelyzed.com

- www.myhandicap.ch

- www.avantidonne.ch

- www.sciparenting.com

\section{Ungeplant schwanger und dann?}

In Alter von 21 Jahren bricht sich Sophie M.* (Name von der Redaktion geändert) bei einem Autounfall den 5. und 6. Halswirbel. Danach ist nichts mehr wie es einmal war. Die Rückenmarkschädigung führt zu einer inkompletten Tetraplegie. Bei dieser Form der Querschnittlähmung sind alle vier Gliedmaßen gelähmt, deshalb braucht Sophie M. * für manche Dinge Assistenz, zum Beispiel für den Transfer aus dem Rollstuhl ins Bett oder auf die Toilette.

„Ich habe mich nach dem Unfall gefragt, ob ich in dem Zustand überhaupt noch mal eine Beziehung haben kann und einen Partner finde, der sich darauf einlässt. Aber über eine Schwangerschaft habe ich mir keine Gedanken gemacht. Die war dann auch nicht geplant. Ich habe meinen Partner kennengelernt, wir waren ein Jahr zusammen und dann war ich schwanger. Es ist einfach passiert. Wir haben uns sofort für das Kind entschieden."

Sophie M.* empfand ihre Schwangerschaft am Anfang als sehr angenehm. Sie hatte dadurch nicht mehr Einschränkungen, als sie sowieso schon hatte. Sie musste nur ein Medikament gegen die Spastik absetzen. „Später war es natürlich sehr anstrengend mit dem Sitzen und dem dicken Bauch. Und weil ich sowieso viel Hilfe brauche. Ich habe vorher immer auf dem Bauch geschlafen, weil ich den ganzen Tag im Rollstuhl sitze. Das ging am Ende auch nicht mehr. Meine Frauenärztin hat sich mit Querschnitt nicht besonders gut ausgekannt. Ich fand mich trotzdem bei ihr gut aufgehoben. Ich hatte nie das Gefühl, dass sie unsicher ist oder nicht weiß, was sie macht. Sie hat dann im Vorfeld direkt gesagt, dass es ein geplanter Kaiserschnitt wird. Ich habe einmal Rücksprache gehalten mit dem Klinikarzt, bei dem ich nach dem Unfall in Behandlung war. Er sagte, wenn ich mich gut fühle und es mir gut geht, sei es eine Schwangerschaft wie jede andere auch. Ich würde im Nachhinein auch sagen: Je weniger Gedanken man sich macht, desto einfacher ist es. Damals gab es auch noch nicht die ganzen Foren im Internet, wo man nachlesen kann, was alles passieren könnte. Da stehen schreckliche Dinge drin und da kann man sich reinsteigern. Man lässt es besser einfach auf sich zukommen.“

\section{Mehr oder weniger ohne Komplikationen}

Auch Sophies* geplanter Kaiserschnitt verlief gut. „Ich habe noch ein zweites Kind bekommen und habe beide
Schwangerschaften als schön erlebt. Die zweite war etwas anstrengender, sie war relativ kurz nach der ersten. Ich habe bei der Geburt ziemlich viel Blut verloren, das hing aber nicht mit dem Querschnitt zusammen. Vielleicht war es, weil die Geburten sehr nah aufeinander folgten, mein zweites Kind so groß war und ich sehr schmal und dünn bin. Aber ansonsten waren beide Schwangerschaften unkompliziert und mir ging es eigentlich immer gut.“

\section{Eine besondere Stillsituation}

Nach der Geburt hatte Sophie M.* eine Hebamme, die keine Erfahrung mit einer Wöchnerin im Rollstuhl mitbrachte. „Die Hebamme ist zu mir nach Hause gekommen, weil ich meine Tochter unbedingt stillen wollte. Das war mir wichtig, nachdem ich sonst schon so viele Einschränkungen hatte. Ich konnte mein Kind zwar im Arm halten, wenn ich im Bett war oder im Stuhl gesessen habe, aber ich konnte es z. B. nicht wickeln und ich konnte viele andere Dinge nicht, daher wollte ich unbedingt stillen. Nach langem Hin und Her hat es endlich geklappt, dann hatte ich aber leider eine Brustentzündung. Während der Antibiotikabehandlung durfte ich nicht stillen, da hat meine Tochter die Flasche bekommen. Danach wollte sie nicht mehr und hat geschrien, wenn sie mich gesehen hat. Bei meinem Sohn war es mir auch nicht vergönnt, der ist beim Stillen eingeschlafen.“

Sophies* Partner gab seine Arbeit auf, um ganz für die Familie da zu sein. „Mein Lebensgefährte hat unsere Kinder gewickelt und gebadet. Ich als Mutter konnte nur danebensitzen. Ich glaube, wenn das eine andere Person gemacht hätte, die mir fremd war, hätte ich das schon komisch gefunden. Es ist schwer genug, wenn es der eigene Partner macht und man nur zuschauen kann.“

\section{Sich emotional abholen lassen}

Sophie M.* ließ sich ihre besondere Situation im Wochenbett nicht groß anmerken, sondern machte das Emotionale mit sich aus. Ob sie sich wohl mehr Unterstützung gewünscht hätte? Heute sagt sie: „Das ist schwierig. Es kann nicht jeder Mensch mit solchen Situationen gut umgehen und es kommt darauf an, wie derjenige, der die Einschränkung hat, sich nach außen gibt. Viele sind sich vielleicht unsicher im Umgang mit Menschen im Rollstuhl. Nicht nur Hebammen, sondern unsere Gesellschaft überhaupt hat ein Problem, mit Menschen umzugehen, die anders sind als-in Anführungszeichen-Normale. Also z. B. Menschen im Rollstuhl oder mit anderen Einschränkungen. Umgekehrt sind manche Leute ganz offen und gehen normal damit um. Dann gibt es Rollstuhlfahrer, die wollen sich nicht helfen lassen. Wenn man als Fußgänger so einem Menschen Hilfe anbietet und dann 
abgewimmelt wird, macht das noch unsicherer. Ich weiß nicht, ob man das ändern kann.“

\section{Von Rollstuhlfahrerinnen lernen}

Wer seine Unsicherheit gegenüber einer ungewohnten Betreuungssituation wahrnimmt, kann sich ihr stellen. Im Krankenhaus wussten die Pflegenden nicht, was aufgrund Sophies* Mastdarmlähmung zu tun ist: „Das manuelle Ausräumen des Stuhlgangs kannte auf der Station niemand. Der Katheder war kein Problem, das mit dem Ausräumen schon. Aber mein Lebensgefährte war beim ersten Kind die meiste Zeit im Krankenhaus dabei. Außerdem hatte ich für vier Stunden am Tag eine Betreuung mit im Krankenhaus, die auch pflegerische Dinge übernommen hat." Rückblickend würde Sophie M.* sich von den Betreuenden wünschen: „Dass sie vielleicht mal die Scheu ablegen und sich eingestehen, nicht alles zu wissen. Man darf auch mal fragen, selbst als Fachkraft. Man kann nicht alles wissen. Es ist besser mal zu fragen, bevor man irgendwas macht, nur um zu überspielen, dass man etwas nicht weiß.“

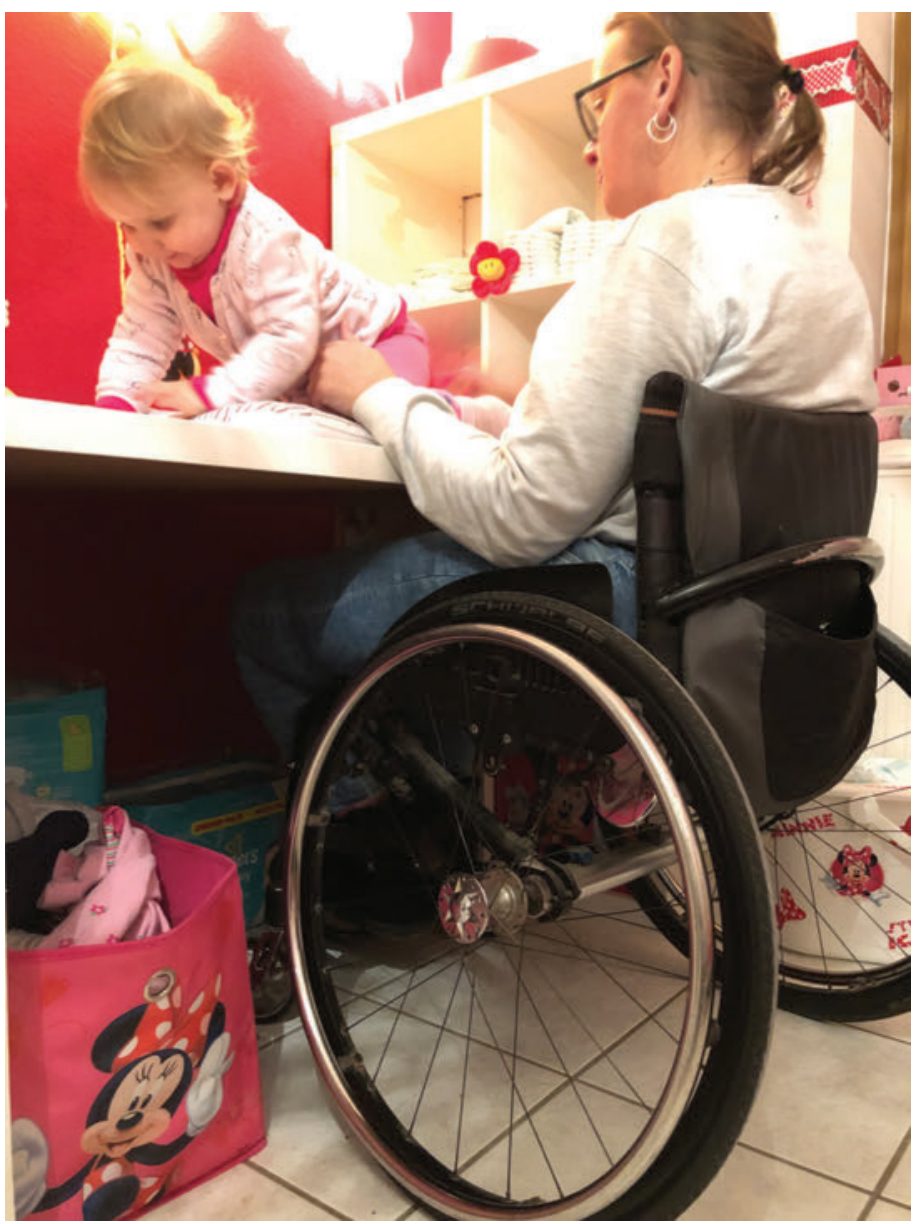

Abb. 2 Egal ob Marke Eigenbau wie dieser Wickeltisch oder gekaufte Einrichtungsgegenstände: Rollstuhlgerechte Möbelstücke sollten unterfahrbar und gut gesichert sein. (Foto: privat)

\section{KONTAKT \\ Bundesverband behinderter und chronisch kranker Eltern}

Der 1999 gegründete Bundesverband behinderter und chronisch kranker Eltern e. V. (bbe) setzt sich für die Interessen, Rechte und mehr Selbstbestimmung von Menschen mit Behinderung oder chronischer Erkrankung im Kontext der Zeugung, Schwangerschaft, Geburt und des Elternseins ein. Auf der Website finden sich viele Informationen zum Download für Eltern und Fachpersonen, z. B. Adressen von Beratungsstellen und Selbsthilfegruppen, Stellungnahmen, Gerichtsurteile, Hilfestellungen für das Schreiben von Anträgen (z. B. für Hilfsmittel und Elternassistenz) sowie barrierefreien Urlaubsunterkünften.

Mütter und Väter mit Querschnittlähmung bedürfen außerdem sinnvoller Hilfsmittel im Umgang mit dem Neugeborenen und Säugling, z. B. bezüglich der eigenen Mobilität mit Kind oder der Anpassung des Schlafplatzes. Allgemein gilt, dass Möbelstücke für das Kind unterfahrbar oder auf Rollen verschiebbar sein sollten. Modelle mit Hilfsmittelnummer können ärztlich verordnet werden [1]. Teils müssen im Umgang mit angepassten Möbeln Sicherheitsmaßnahmen ergriffen werden. Mit einem Rollstuhladapter kann der Säugling z. B. direkt am Rollstuhl transportiert werden. Bei Autokindersitzen erleichtern schwenkbare Modelle das Hineinsetzen, Anund Abschnallen des Kindes. Teils können Hilfsmittel wie Transporthilfen ausgeliehen werden. Mehr Infos zu käuflichen oder selbst hergestellten Hilfsmitteln auf der Website des bbe:

- www.behinderte-eltern.de

\section{"Das geht schon irgendwie“}

Laura P.* (Name von der Redaktion geändert) war 19 Jahre alt, als sie bei einem Autounfall einen Brustwirbelbruch zwischen BK 5 und 7 erlitt, der zu ihrer Querschnittlähmung führte. Heute hat sie gelernt, mit der Lähmung von Beinen, Blase und Darm umzugehen und managt selbstbewusst ihre mittlerweile sechsköpfige Familie. Aber sie erinnert sich gut, was sie direkt nach dem Unfall dachte: „Als sie mich aus dem OP geholt haben, war meine erste Frage, ob das noch funktioniert mit Kindern." Tatsächlich hatten sie und ihr Partner einen Kinderwunsch und setzten diesen alsbald in die Tat um. Auf die Zeit des Mutterwerdens blickt sie positiv zurück: „Mit 22 Jahren habe ich die Pille abgesetzt und wurde auf natürlichem Wege schwanger. Ich dachte immer, das geht schon irgendwie. Meine Schwangerschaften waren unkompliziert. Mein Ältester wird jetzt 19 Jahre alt und die Jüngste ist 11 Monate.“ 


\section{Expertin im Rollstuhl}

Wie die meisten Schwangeren nahm Laura P.* die drei regulär im Mutterpass vorgesehenen Ultraschalluntersuchungen in der Schwangerschaft wahr. „Das finde ich für uns eigentlich zu wenig“, sagt Laura P.* mit Blick auf ihre Rückenmarkschädigung. „Weil wir vielleicht die Schmerzen, die Kindsbewegungen und die Wehen nicht so merken wie jede andere Frau. Ich habe immer mit dem Handy meinen Bauch gefilmt, um die Kindsbewegungen zu sehen. Die Kleine hat sich oft um die eigene Achse gedreht. Wenn ich dann zwei bis drei Tage keine Bewegung sehe, beunruhigt das schon sehr. Deshalb muss ich vielleicht einmal mehr Ultraschall machen lassen. Mein Frauenarzt war kulant und hat gesagt, wenn es mich beunruhigt und ich deswegen Stress habe, ist das fürs Kind nicht gut. Da hat er mehrmals zwischendurch einen Ultraschall gemacht und ich war beruhigt."

Mit jeder Schwangerschaft wurde Laura P.* mehr Expertin über die Vorgänge in ihrem Körper. Mehr Vorsorgen als andere brauchte sie bald nicht mehr. „Bei meinen ersten Kindern hat man es auch noch nicht so genau genommen. Heute wird die regelmäßige Gewichtskontrolle empfohlen. Aber welcher Frauenarzt hat schon eine Sitzwaage? Damals haben sie mein Gewicht geschätzt oder gar nicht aufgeschrieben. Bei meinem vierten Kind, das ich mit 39 Jahren bekommen habe, musste ich zum ersten Mal Heparin spritzen, weil wohl über die Jahre ein Faktor5-Leiden bei mir aufgetaucht ist. Es war eine Vorsichtsmaßnahme meines Frauenarztes im Hinblick aufs Alter. Mein jetziger Gynäkologe kannte sich nicht aus mit Querschnitt, hat es aber locker gesehen, weil ich schon drei Kinder hatte. Ich bin sowieso der Meinung, dass ich meinen Körper am besten kenne. Er hat es aber gut gemacht und wenn er nicht weiterwusste, hat er mich ins Krankenhaus überwiesen. Die kennen sich mit Schwangerschaft und Querschnitt allerdings auch nicht wirklich aus ..."

Die Hebamme, die Laura P.* bei ihrem dritten Kind betreute, hatte keine Erfahrung mit einer querschnittgelähmten Schwangeren. Was die routinierte werdende Mutter achselzuckend hinnahm, könnte manche Erstgebärende verunsichern: „Sie hat eine große Aufregung um die Geburt verbreitet und angefangen aufzuzählen, was ich angeblich alles nicht könnte. Da hab ich gesagt: , Stop! Ich habe zwei Kinder geboren, schneller als manche andere Frau. Die sind auf ganz natürlichem Weg da rausgeschliddert. Warum soll ich jetzt Angst haben oder einen auf Vierfüßlerstand machen?` Meine Erfahrung ist: Wir müssen den Ärzten und Hebammen manchmal erklären, wie das bei uns ist und was wir brauchen. Ihnen fehlt die Erfahrung, denn Frauen im Rollstuhl sind ja noch selten im Kreißsaal.“ 


\section{Selbstbestimmt gebären}

Drei von vier Kindern brachte Laura P.* völlig komplikationslos auf vaginalem Wege zur Welt. „Den dritten wollte ich per Kaiserschnitt holen lassen, weil B-Streptokokken nachgewiesen worden sind und das Risiko zu groß war, dass sich das Kind ansteckt. Ich wollte ihm auch die Antibiotikagabe ersparen. Ich habe den Kaiserschnitt ohne Narkose oder PDA machen lassen. Die Ärzte befürchteten erst, dass ich Schmerzen haben würde beim Schneiden. Das war aber nicht der Fall. Die Spastik nahm stark zu beim Schneiden - ein Ziehen und Zucken ging von den Beinen bis in die Bruchhöhe, das war nicht so spaßig. Aber es hat nicht weh getan. Ich habe zwar den Druck gespürt, als sie gedrückt und geschoben haben, empfand aber keinen Schmerz. Ich war in Augenkontakt mit dem Chirurgen, der immer gefragt hat, ob alles gut ist. Die Anästhesisten standen auch bereit und wenn irgendwas gewesen wäre, hätten sie mir eine Narkose gegeben.“

Bei allen drei Vaginalgeburten hatten es Lauras* Kinder eilig: „Beim ersten habe ich zweieinhalb Stunden gebraucht. Da haben die Hebammen von oben ein bisschen mitgedrückt. Das war aber okay für mich. Klar, angenehm ist das nicht, wenn einer auf meinem Bauch hantiert. Aber ich denk mir immer: ,Was muss, das muss. 'Beim zweiten Kind hatte ich Wehen, als ich in den Kreißsaal kam. Die Hebamme wollte noch einen Wehenschreiber dranmachen. Da sagte ich: ,Das brauchen Sie nicht, das Kind ist gleich da.' Als ich die Hose runtergemacht habe, konnte man die Schädeldecke schon sehen. Die Hebamme hat die Fruchtblase aufgemacht und dann habe ich zweimal gepresst. Das kann ich zwar nicht so stark wie eine Frau ohne Querschnittlähmung, aber es geht. Insgesamt hat der Kleine nur 12 Minuten gebraucht, meine jüngste Tochter nur 5 Minuten. Bei ihr bin ich eingeleitet worden, weil ich zum Schluss hin zu wenig Fruchtwasser hatte. Wenn ich Wehen hatte, machte sich das normal mit kompletter Gänsehaut von Kopf bis Fuß bemerkbar. Nach der Einleitung hat sich bei jeder Wehe der ganze Bauch komplett zusammengezogen, das war unangenehm, aber ich hatte keine Schmerzen. Als ich endlich in den Kreißsaal kam, waren es nur noch 20 Sekunden zwischen den Wehen. Die Hebamme wollte noch den Katheder ziehen, die Ärztin noch Herztöne messen, aber da war das Kind schon da. Die hatten wohl gedacht, ich brauche länger, weil ich querschnittgelähmt bin.“

Laura P.* trägt tagsüber einen Dauerkatheder mit Beinbeutel, weil ihre Blase seit dem Unfall nur noch kleine Mengen Urin hält (ca. $250 \mathrm{ml}$ ) und das sonst sehr unangenehm werden kann kann. „Ich war mal auf einem Ausflug und plötzlich ging es los, als wäre die Fruchtblase geplatzt. Ich war nass bis in die Kniekehlen und musste den ganzen Tag so rumfahren. Dann habe ich mit meinem Querschnittarzt gesprochen und entschieden: Dauerkatheder. Den mache ich morgens selbst rein und ziehe ihn abends wieder, sodass die Blase über Nacht selbst arbeiten kann. Das funktioniert gut."

\section{Mut zur Vaginalgeburt}

Laura P.* würde auch anderen Frauen im Rollstuhl raten, auf physiologischem Wege zu gebären, sofern keine Kontraindikation vorliegt: „Ein Kaiserschnitt ist schon eine große OP. Und eigentlich sind wir dafür gemacht, dass die Kinder da unten rauskommen, wo sie auch reingekommen sind“, sagt sie augenzwinkernd. Natürlich könne man nie wissen, wie die Geburt verläuft, räumt die Mutter von vier Kindern ein. Doch spiele die innere Einstellung vielleicht eine Rolle, vermutet sie: „Wenn jemand mit Angst in den Kreißsaal kommt und denkt, das funktioniert bei ihr sowieso nicht, sie kann das nicht-dann ist womöglich der Kaiserschnitt für diese Frau die bessere Wahl. Aber als Rollstuhlfahrerin muss sie sich im Klaren darüber sein, dass sie viel sitzt. Und ob die Narbe bei jeder Frau so gut heilt, wenn da den ganzen Tag Spannung drauf ist, weiß ich nicht.“

\section{Die ersten Tage mit Baby}

Als Herausforderung erlebte Laura P.* die ersten Tage im Wochenbett. „Für Rollstuhlfahrerinnen ist es extrem schwierig, mit den Nachblutungen umzugehen. Aber ich hatte zwei tolle Pflegerinnen auf der gynäkologischen Station, die mich mit Bettpfanne im Bett gespült haben und eine frische Vorlage reingemacht haben-das hat hervorragend funktioniert. Sie haben auch ein elektrisches Bett organisiert, das normal nur Privatpatienten bekommen. Das Spülen hätte ich alleine auf einer Toilette nicht hinbekommen.“

Lange im Bett hielt es Laura P.* nach ihren Geburten nicht. „Ich bin drei bis fünf Stunden nach der Geburt wieder raus aus dem Bett, weil ich ja ein Kind hatte. Die Kleine wollte die Flasche, sie wollte einen sauberen Popo ... Ich klingle auch nicht bei jedem Pups. Außer mitten in der Nacht, wenn die Kleine die Flasche will. Dann geht das einfach schneller.“

\section{Familienmanagerin im Rollstuhl}

Das Familienleben zu Hause meistert Laura P.* wie jede andere Mutter. Nur eben im Rollstuhl. „Das geht ganz gut. Ich bin die ganze Woche alleine, mein Mann arbeitet auf Montage. Ich habe meinen Haushalt und meine Kinder. Klar, dauert vieles länger als bei anderen. Manchmal hab ich auch keinen Bock, den Haushalt zu machen. Aber das geht anderen genauso. Es ist sicher nicht immer toll oder einfach. Aber in welcher Familie ist es das schon? Das hat nichts mit dem Querschnitt zu tun. Dass man mal außer sich ist wegen etwas oder genervt, wenn die Nacht anstrengend war, das haben andere Mütter auch.“ 
Neben der Gelassenheit, mit der Laura P.* ihr Familienleben managt, erleichtern ihr einige Hilfsmittel das Muttersein im Rollstuhl. Den Wickeltisch, den sie mit ihrem Rollstuhl gut unterfahren kann, haben sich die Eltern selbst gebaut und an der Wand gesichert. „Auf einer Wickelkommode aus dem Babymarkt könnte ich mein Kind gar nicht wickeln. Manches ist bei uns schon anders. Den meisten fehlt ja - je nach Bruchhöhe - die Rücken- und Bauchmuskulatur. Ich muss einen festen Halt haben, wenn ich die Kleine irgendwo raushole, dass sie mir nicht runterfällt. Wir achten bei Möbeln darauf, dass ich sie so wenig wie möglich heben und dass ich nur kurze Distanzen überbrücken muss.“ Zum Spazierenfahren mit ihrer Tochter benutzt Laura P.* ein Fahrrad mit Elektromotor, das es ihr ermöglicht, den Kinderwagen mit einer Hand zu führen. „Das können sich viele nicht vorstellen, aber es funktioniert.“

\section{Fazit}

Alles in allem zeigen diese drei Frauen mit Handicap exemplarisch, dass für sie Mutterwerden und Muttersein eine Herausforderung ist, die sich managen lässt. Aber, um es mit Lauras Worten zu sagen: „Bei welcher Mutter ist es das nicht?"

\section{ZUM WEITERLESEN}

\section{Infobroschüren}

- Ideenwettbewerb Barrierefreie Kinder- und Babymöbel: Im Jahr 2003 wurde vom bbe e. V. anlässlich des Jahres der Behinderung ein Ideenwettbewerb ausgerufen, zu dem Vorschläge für barrierefreie Möbel eingereicht werden konnten. Neben Lösungen für die Zielgruppe der Eltern mit Sehbehinderung wurden auch solche für rollstuhlfahrende Eltern gesucht. Die Broschüre zeigt Vorschläge für Transporthilfen für rollstuhlfahrende Eltern, angepasste Wickeltische und Babybetten sowie Bezugsquellen dieser besonderen Möbel.

- Kinderanhänger für E-Rollstuhl gesucht - Ratgeberreihe Band 2: Dieser Ratgeber enthält weitere Ideen für barrierefreie Möbel für den Familienalltag für betroffene Eltern und auch Berufsgruppen, die mit den Eltern arbeiten. Hebammen werden explizit als Adressaten der Broschüre benannt.
- Elternassistenz_Unterstützung für Eltern mit Behinderung und chronischen Erkrankungen. Ratgeber für die Beantragung und Organisation personeller Hilfen zur Betreuung und Versorgung der Kinder.

\section{Autorinnen / Autoren}

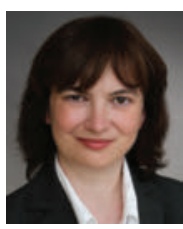

Anja Stamm ist freie Redakteurin mit dem Schwerpunkt Gesundheitskommunikation in Frankfurt am Main.

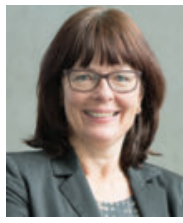

Prof. Dr. rer. medic. Ute Lange ist Hebamme und Professorin für Hebammenwissenschaft an der Hochschule für Gesundheit Bochum. (Foto: hsg Bochum)

Korrespondenzadresse

hsg Bochum | Hochschule für Gesundheit Studienbereich Hebammenwissenschaft Prof. Dr. Ute Lange

Gesundheitscampus 6-8

44801 Bochum

E-Mail: ute.lange@hs-gesundheit.de

\section{Literatur}

[1] Richtlinie des Gemeinsamen Bundesausschusses über die Verordnung von Hilfsmitteln in der vertragsärztlichen Versorgung(Hilfsmittel-Richtlinie/HilfsM-RL) von 2011. zuletzt geändert 2017. https://www.g-ba.de/downloads/62-492-19 22/HilfsM-RL_2017-07-20_iK_2019-09-13.pdf

\section{Bibliografie}

DOI https://doi.org/10.1055/a-1084-5160

Die Hebamme 2020; 33: 31-37

(C) Georg Thieme Verlag KG Stuttgart · New York ISSN 0932-8122 